\title{
Smart Nanocarriers and Drug Delivery: Soft Interaction and Colloidal Stability in Complex Biological Media
}

\author{
Dr. Domenico Lombardo \\ CNR-IPCF, Italy
}

\begin{abstract}
In recent years, novel approaches for the development of nanocarriers and nano-formulations for the efficient transport of drug molecules in living systems offer a wide range of biotechnology applications. A wide range of smart integrated nanosystems have proven their effectiveness for various types of biomedical tasks, including stimuli-responsive liposomes, polymeric and metal nanoparticles, silica and hybrid (organic/inorganic) nanostructures [1,2].

However, despite the remarkable developments of recent synthetic methodologies, most of all nanocarrier's action is associated with a number of unwanted side effects that diminish their efficient use in nanomedicine [3, 4]. The major limitation in the use of such versatile and smart drug delivery systems is connected with their limited colloidal stability arising from the interaction with the complex environment and multiform interactions established within the specific biological media. The complex microenvironment in living systems strongly affects the functionality of nanomaterials, and may compromise the design goals of nanocarriers.

We discuss the main factors that influence the nanocarriers colloidal stability during their interaction with biological media during drug delivery applications [5-7]. We also discuss some strategies that can be developed to overcome the biological barriers, and how these approaches have stimulated the development of advanced drug delivery systems.
\end{abstract}

\section{References}

[1] G Chen, I Roy, C Yang, et al., Chemical Review, 116, 2826 (2016)

[2] D Lombardo, MA Kiselev, MT Caccamo, Journal of Nanomaterials, 3702518, (2019)

[3] S. Wilhelm, A. J. Tavares, Q. Dai, et al., Nature Reviews Materials, 1, 16014 (2016)

[4] D Lombardo, P Calandra, D Barreca, S Magazù, M Kiselev, Nanomaterials, 6, 125, (2016)

[5] D Lombardo, P Calandra, E Bellocco, et al. Biochimica et Biophysica Acta (BBA)-Biomembranes, 1858, 2769, 2016)

[6] D Barreca, G Laganà, G Toscano, P Calandra, MA Kiselev, D Lombardo, Biochimica et Biophysica Acta (BBA)-

General Subjects, 1861, 3531, (2017)

[7] D. Lombardo, P. Calandra, S. Magazù, et al., Colloids and Surfaces B: Biointerfaces, 170, 609, (2018).. 